\title{
Modifying Threat-related Interpretive Bias in Adolescents
}

\author{
Elske Salemink • Reinout W. Wiers
}

Published online: 25 May 2011

(C) The Author(s) 2011. This article is published with open access at Springerlink.com

\begin{abstract}
Socially anxious feelings sharply increase during adolescence and such feelings have been associated with interpretive biases. Studies in adults have shown that interpretive biases can be modified using Cognitive Bias Modification procedures (CBM-I) and subsequent effects on anxiety have been observed. The current study was designed to examine whether the CBM-I procedure has similar effects in adolescents. Unselected adolescents were randomly allocated to either a positive interpretation training $(n=88)$ or a placebo-control condition $(n=82)$. Results revealed that the training was successful in modifying interpretations and effects generalized to a new task. The interpretive bias effects were most pronounced in individuals with a threat-related interpretive bias at pre-test. No effects on state anxiety were observed. The current findings are promising with regard to applying bias modification procedures to adolescents, while further research is warranted regarding emotional effects.
\end{abstract}

Keywords Interpretive bias - Cognitive bias modification . Anxiety. Adolescence

\section{Introduction}

Social anxiety and social phobia have a great impact on daily life functioning in youth; individuals feel anxious in social situations and the avoidance of a broad range of situations causes detriments to children's social and

E. Salemink $(\square) \cdot$ R. W. Wiers

Department of Developmental Psychology,

University of Amsterdam,

Roetersstraat 15,

1018 WB Amsterdam, The Netherlands

e-mail: E.Salemink@uva.nl academic functioning (Woodward and Fergusson 2001). Specifically, the adolescent period is characterized by a strong increase in fear of negative social evaluation relative to a consistent decrease in general fears (Westenberg et al. 2007). Furthermore, social phobia, the clinical form of social anxiety, typically has its onset in adolescence (age of onset between 10 and 16.6 years, Wittchen and Fehm 2003). The course of social phobia is chronic and it has been shown that the strongest predictor of recovery is late age of onset of social fears (DeWit et al. 1999), underscoring the need for (preventive) interventions directed at social fears in children and adolescents.

Cognitive models of anxiety propose that the tendency to interpret ambiguous information as threatening plays a causal role in the etiology and maintenance of pathological anxiety (Beck et al. 1985). There is overwhelming evidence in adults that such threat-related interpretive biases and anxiety are related (Mathews and MacLeod 1994) and such evidence is accumulating in children and adolescents. Hadwin et al. (1997), for example, revealed that anxiety is associated with threat-related interpretations in a nonselected (i. e. selection was not based on specific criteria) sample of children (7 to 9-years-old). Bögels and Zigterman (2000) showed that in a clinical sample, children and adolescents with an anxiety disorder (separation anxiety, social phobia, or generalized anxiety) interpreted ambiguous situations as more negative than children with externalizing problems (clinical control group) or a non-clinical control group (mean age entire sample $=12.5$ years). The role of a negative interpretive bias regarding social information in socially anxious adolescents has also been demonstrated; high socially anxious adolescents (mean age 13.6 years) interpreted short scenarios regarding social interactions as more negative than adolescents with an average level of social anxiety (Miers et al. 2008). This relationship between 
threat-related interpretive bias and childhood anxiety tends to be moderated by age; interpretive bias seems to be associated with anxiety in older children (above 11 years), but less so in younger children (Cannon and Weems 2010; Weems et al. 2001). The evidence for the influence of gender on the relationship between interpretive bias and anxiety is more mixed. While several studies have shown that gender did not moderate the link between interpretive bias and anxiety (Leitenberg et al. 1986; Weems et al. 2001, 2007), a recent study showed that interpretive bias was significantly related to anxiety disorder status for females, but not for males (Cannon and Weems 2010).

It is assumed that this threat-related interpretive bias is not an incidental epiphenomenon of anxiety, but that it plays a causal role in the maintenance and the development of an anxiety disorder (Beck et al. 1985; Muris and Field 2008). While correlational studies presented above confirmed the relationship between interpretive bias and anxiety, studies with a pure experimental design are necessary to confirm the causal relationship. In adults, there is now sound empirical evidence that a threat-related interpretive bias can causally influence anxiety. Mathews and Mackintosh (2000) developed a Cognitive Bias Modification procedure to modify interpretations (CBM-I) in adults. Participants read short ambiguous social scenarios that ended with a word fragment requiring solution. Correct solution of the fragment disambiguated the story either positively or negatively, depending on the assigned condition. In this way, participants in the positive CBM-I condition were trained to impose positive interpretations on ambiguous information, while participants in the negative condition were trained to interpret such information in a negative way. The computer program proved to be successful in modifying interpretive biases and subsequent effects on anxiety were observed, providing evidence consistent with a causal link between interpretive bias and anxiety. It appears to be a robust finding, as the effects have been replicated several times in adult samples of unselected individuals (Holmes et al. 2006; Salemink et al. 2007b) and effects have been observed on interpretive bias $24 \mathrm{~h}$ later (Yiend et al. 2005). The ability to modify threat-related interpretive biases with effects on anxiety not only has important theoretical implications (causality), but also important clinical implications. In adults, it has been shown that training individuals with high levels of anxiety to interpret ambiguous information in a positive way using CBM-I reduced their negative interpretive bias and associated self-reported anxious feelings (Mathews et al. 2007; Murphy et al. 2007; Salemink et al. 2009).

There are three recent studies that have examined modification of interpretive bias in children (Muris et al. 2008, 2009; Vassilopoulos et al. 2009). Children in Muris et al.'s (2008) study completed a computer game "Space odyssey" that consisted of an imaginary journey to an unknown planet. During the journey they encountered different ambiguous situations that can be interpreted in a positive or negative way. Children in the positive training condition received reinforcement for choosing positive outcomes, whereas children in the negative training condition received reinforcement for choosing negative outcomes. Results revealed that interpretive bias was successfully modified using this CBM-I procedure and that increase in negative interpretations was particularly pronounced in children with high levels of anxiety (Muris et al. 2008). These CBM-I effects in children were later replicated, without replicating the moderating effects of pre-training anxiety (Muris et al. 2009). In addition, training affected avoidance tendencies; children in the negative training condition placed themselves further away from new situations and objects on a map depicting the new planet. This suggests generalization of the trained interpretive bias, which is interesting, as there are inconsistent findings regarding generalization to other tasks and domains in adults (Salemink et al. 2007b, 2010a). Note however that effects on anxiety were not investigated and changes in interpretations were quite small (partial $\eta^{2} \leq 0.05$ ). The latter could possibly be due to the relative young age of these children (first study: 10.0 years; second study: 10.7 years), as negative interpretive biases appear to be related to anxiety in older children ( $>11$ years, Cannon and Weems 2010). Vassilopoulos et al. (2009) selected high socially anxious children, who were trained over three sessions to endorse positive rather than negative interpretations. Again it was shown that CBM-I training was successful in changing interpretations, and, more importantly, children in the training condition showed reduced trait social anxiety. Taken together, these findings in children are promising with regard to the modification of interpretations and effects on anxiety.

The goal of the current study was to examine whether CBM-I can modify interpretations and anxiety in adolescents. CBM-I is a computerized training to modify threat-related interpretive biases that has proven to be effective in adults and promising in children. In the current study, CBM-I was applied to adolescents, as adolescence is characterized by a sharp increase is social anxiety (Westenberg et al. 2007) and specifically in this age period, anxiety is associated with threat-related interpretive biases (Cannon and Weems 2010). The first aim was to examine whether CBM-I can modify threat-related interpretations in adolescents. Therefore, adolescents were randomly allocated to either a positive CBM-I condition to learn to interpret ambiguous social information in a more positive way or to a placebo-control condition. The second aim was to examine generalization of the modified interpretive bias to a new task containing new stimuli. A recognition task was presented before and after CBM-I to examine generalization of the trained 
interpretive bias. The third aim was to examine factors that might influence CBM-I effects. As previous research with children has shown that level of anxiety could moderate the effects of training (Muris et al. 2008), pre-training levels of trait anxiety and interpretive bias were assessed. The final aim was to examine the effects of CBM-I on state anxiety.

It was hypothesized that 1) following training, adolescents in the positive condition would interpret ambiguous information significantly more positive and less negative than adolescents in the placebo-control condition, when controlling for age and gender. It was also hypothesized that 2) trained interpretive bias would generalize to a new task and 3) that pre-training level of trait anxiety and interpretive bias would moderate the effects of training. Lastly, 4) we predicted that adolescents in the positive training condition would feel less anxious following training than adolescents in the placebo-control condition.

\section{Method}

\section{Participants}

The current study had no inclusion or exclusion criteria and all adolescents from a class level were invited to participate. The sample consisted of 170 adolescents (79 boys and 91 girls) drawn from a public secondary school (two education levels: "havo": senior general secondary education and "vwo": pre-university education) in the Netherlands. Adolescents ranged in age from 14-16 years $(M=14.5, S D=0.5)$ and $98.2 \%$ was born in the Netherlands. The study was approved by University of Amsterdam's Ethical Committee (Department of Developmental Psychology) and passive informed parental consent was obtained for all participating adolescents.

Participants were randomly allocated to either the positive CBM-I $(n=88)$ or the placebo-control $(n=82)$ condition. For ethical reasons, the positive training condition was not compared to a negative training condition. Due to technical difficulties (losing connection with the server that ran E-prime), the computer program sometimes stopped, resulting in lost data. Twelve participants were lost between the start of the experiment and the end of CBM-I, resulting in 158 adolescents who finished the complete CBM-I program and the post-training state anxiety assessment (81 participants in the positive training condition and 77 in the placebo control condition). In total, 144 adolescents completed the second recognition task, thus generalisability effects were analyzed for these 144 participants ( 75 participants in the positive training condition and 69 in the placebo control condition). ${ }^{1}$

\footnotetext{
${ }^{1}$ Analyses regarding reaction time and state anxiety were repeated on the smaller complete set of 144 participants. The same pattern of results was observed, albeit not always reaching significance, presumably due to reduced power.
}

Materials

$C B M-I$ A social scenario-based training was used to modify interpretive bias (Mathews and Mackintosh 2000). Dutch scenarios that were successfully used in previous experiments with non-selected adults (Salemink et al. 2007b) were modified to reflect social situations that are relevant for young adolescents. A small pilot study confirmed the age appropriateness of the scenarios. The training consisted of five blocks of 10 scenarios that were presented on a personal computer. In each block, participants in the positive CBM-I group received eight positive modification scenarios and two probe scenarios, while participants in the placebo-control condition received four valenced scenarios (two positive and two negative), four neutral scenarios, and two probe scenarios. The modification scenarios were created to train participants to make a positive emotional interpretation of an initially ambiguous social situation. Each scenario consisted of three lines that were ambiguous in terms of valence. In the final sentence, a word was left out of the sentence. After disappearance of the scenario, the omitted word was presented as a word fragment and disambiguated the scenario in a positive way. Participants were instructed to press a spacebar when they felt they knew what the word was, and then to press the key corresponding to the first missing letter. This ensured that the time taken to respond to the word was not contaminated by variance in the time taken to locate the correct letter key. The computer program only continued when a correct response was given. Following the solution of the word fragment, a comprehension question appeared that could extend to future emotional implications beyond those actually stated in the scenario. Participants answered the question by pressing $\mathrm{Y}$ for yes and $\mathrm{N}$ for no. They subsequently received feedback (correct vs. wrong answer) to reinforce the interpretation. An example of a modification scenario, with the completed word fragment in parentheses, is as follows:

You are working on a school assignment with some other children at your house. You turn on your favorite music and based on their reactions, you understand that they ...... your choice of music.

1-ke (like)

Then the comprehension fragment followed:

Did your friends approve of your choice of music?

Participants in the placebo-control condition received two positive and two negative scenarios. In the negative variant of the previous example, participants would for example receive 'disl-ke' (dislike). Participants in this placebocontrol condition also received neutral scenarios that had 
no emotional content, nor did they contain any ambiguity. An example is as follows:

After the summer holidays, you are going back to school.

As you arrive, you cannot help noticing

that the outside of the school has been

pa-nt-d (painted)

Has your school changed its appearance?

Test of Interpretive Bias: Reaction Times Probe scenarios were included in the training procedure to assess change in interpretation style. Probe scenarios were also three-lines in length and described an initially ambiguous situation. However, both groups received the same probe scenarios with the same word fragments; one positive and one negative valenced word fragment per block, resulting in a total of five positive and five negative probes. The speed of correctly resolving these probe word fragments was the dependent measure (CBM-I effects on interpretive bias).

Test of Generalization: Recognition Test To assess generalization of modified interpretive bias to a new task containing new scenarios, participants completed a recognition task before and after CBM-I. Each task consisted of two parts with part two being the actual assessment of interpretive bias. In part one, seven ambiguous social scenarios were presented (different scenarios were used for the pre- and post-training test). Each scenario had a title and consisted of three lines. A word was missing in the final sentence that was presented as a word fragment after pressing the space bar. Again, participants were asked to complete the fragment as quickly as possible, yet the valence of the story remained ambiguous. Afterwards, a comprehension question with relevant feedback appeared on the screen and participants were asked to answer the question.

In the second part of the recognition test, participants saw the title of the ambiguous scenario, this time together with two interpretations of the scenario; a positive interpretation and negative interpretation. Participants rated each sentence independently for its similarity in meaning to the original scenario (presented in part one). A 4-point scale was used, ranging from 1 (very different) to 4 (very similar). Endorsement of positive versus negative interpretations in this second part was the dependent measure (generalization of CBM-I effects to the recognition task).

Anxiety Assessment As previous studies with adults observed effects of interpretation training on state anxiety (a transitory anxious state) assessed with the Spielberger State Trait Inventory (STAI, Mathews et al., 2007; Mathews and Mackintosh 2000), state anxiety in the current study was assessed with the Dutch State version of the State Trait
Anxiety Inventory for Children (ZBV-K, Bakker et al. 1989; STAI-C, Spielberger 1973). Pre-training level of trait anxiety (the stable tendency to react with anxious feelings to a stressful situation) was assessed with the Dutch Trait version of the STAI-C (Bakker et al. 1989; Spielberger 1973).

Procedure

The experiment took place in the school's computer room. Participants were informed about the procedure and that specific task instructions would appear on the computer screen. The computer-program (E-prime version 2.0) presented the STAI-C trait and state questionnaire first. Participants then completed the first recognition task. ${ }^{2}$ Afterwards, they were randomly assigned to either the positive CBM-I condition or the placebo-control condition. Participants were specifically instructed to imagine themselves in each of the presented situations. An option for rest was given after each block, resulting in four short breaks. After CBM-I, participants completed the STAI-C state for the second time and completed the second recognition task. At the end of testing, participants were debriefed. The session took approximately $45 \mathrm{~min}$.

\section{Results}

\section{Correlational Analyses}

We examined the relationship between interpretive bias, anxiety, gender, and age (assessed before CBM-I) using correlational analyses $(n=158$, see Table 1$)$. A pre-training interpretive bias index was calculated by subtracting mean similarity scores of positive interpretations from mean similarity scores of negative interpretations, with positive scores indicating a threat-related interpretive bias. Results indicated that interpretive bias scores were positively associated with trait and state anxiety, revealing that, consistent with previous findings, higher levels of anxiety were associated with a stronger threat-related interpretive bias. In addition, gender was significantly correlated with trait and state anxiety; females reported more state and trait anxious feelings. Furthermore, gender was also significantly correlated with pre-training interpretive bias; girls had stronger threat-related interpretations than boys. When directly comparing male and female adolescents, it was shown that female adolescents felt significantly more

\footnotetext{
$\overline{{ }^{2} \text { A subgroup }}(n=67)$ received a colour-word Stroop task before and after training. This task is not part of the presented study and will not be reported. Stroop task (performed yes vs. no) was added as an additional between-subjects factor to the analyses to examine whether this interacted with the main findings. It did not, $p$-values $>0.36$.
} 
Table 1 Correlations, means, and standard deviations for interpretive bias, anxiety, gender and age at pre-training assessment $(n=158)$

${ }^{*} p<0.05,{ }^{* *} p<0.01$

\begin{tabular}{l} 
\\
\hline 1. Interpretive bias \\
2. Trait anxiety \\
3. State anxiety \\
4. Gender \\
5. Age \\
$M$
\end{tabular}

$S D$
1.

$0.31 * *$
$0.25 * *$
$-0.20 *$
0.06
0.17

0.7
2.

$0.52 * *$
$-0.30 * *$
-0.11
29.7

6.2
3.

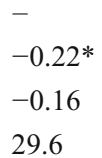

0.07

81 female /

77 male
5.

3.7
14.5

0.5 state and trait anxious than males, respectively $t(149)=2.8$, $p=0.006, d=0.5$ (state anxiety girls $M=30.4, S D=3.7$ vs. boys $M=28.8, S D=3.6)$ and $t(156)=4.0, p<0.001, d=0.6$ (trait anxiety girls $M=31.5, S D=6.3$ vs. boys $M=27.8$, $S D=5.5$ ). Furthermore, female adolescents also had a significantly stronger negative interpretive bias compared to males, $t(150)=2.4, p=0.02, d=0.4$ (girls $M=0.30, S D=0.6$ vs. boys $M=0.04, S D=0.7$ ).

\section{Effects of CBM-I on Interpretive Bias (Hypothesis 1)}

Regarding reaction time analyses, reaction time data were excluded if the response to the probe word fragment $(0.3 \%)$ or comprehension question (13.4\%) was incorrect or if the latency was less than $200 \mathrm{~ms}$ (1.1\%). One participant lost all the reaction time data for the negative probes and was excluded from the analyses. Participants in the positive and placebocontrol condition did not differ significantly in the percentage of incorrect responses to probe word fragments, $t(155)=1.0$, $p=0.30\left(M_{\text {positive }}=0.1, S D=1.1\right.$ vs. $\left.M_{\text {placebo }}=0.4, S D=1.9\right)$ nor to probe comprehension questions, $t(155)=-0.4, p>0.50$ $\left(M_{\text {positive }}=13.9, S D=13.8\right.$ vs. $\left.M_{\text {placebo }}=13.0, S D=13.7\right)$. Exploration of the distribution of the reaction time variables (boxplot and absolute z-scores) revealed 5 outliers that were removed from the analyses (z-score $>2.58$ ), resulting in 152 participants with 77 participants in the positive training condition and 75 in the placebo control condition.

To test whether the CBM-I procedure was effective in influencing interpretations in adolescents, the reaction time data was subjected to a 2 (Condition: positive training vs. placebo control condition) x 2 (Valence probe: positive vs. negative) $x 2$ (Gender) mixed model ANCOVA with age as the covariate. Gender was included as an additional between-subjects variable as it is known from the literature that girls are more vulnerable for the development of internalizing problems (Craske 2003) and as gender was correlated with both interpretive bias and anxiety in the current study. Age was added as a covariate to the analysis as age differences in the relationship between anxiety and interpretive bias have been observed (Cannon and Weems 2010). This analysis revealed the predicted Condition $x$
Valence interaction effect, $F(1,147)=10.9, p=0.001, \eta_{\mathrm{p}}{ }^{2}=$ 0.07 (for means and standard deviations, see Table 2). Positively trained adolescents were quicker to solve positive than negative word fragments $t(76)=-4.3, p<0.001, d=0.5$, while adolescents in the placebo-control condition did not differ significantly in their responses to positive and negative solutions of the ambiguous scenario, $t(74)=0.4, p>0.50$, $d=0.05$. Direct comparison of the two conditions revealed that adolescents trained to interpret information positively were significantly faster to complete positive probes compared to individuals who followed the placebo-control training, $t(150)=2.6, p=0.01, d=0.4$. The groups did not differ significantly from each other in solving negative probes, $t(150)=-0.1, p>0.50, d=0.02$. No other significant main or interaction effects, including gender and age ( $p$ values $>0.13$ ) were observed. Thus, it was shown that the cognitive bias modification procedure was effective in modifying interpretations of ambiguous social situations in adolescents.

\section{Generalization of CBM-I (Hypothesis 2)}

In the recognition task data, five participants were identified as outliers and excluded, resulting in $n=139$ participants who completed both the pre- and post-training recognition task $(n=73$ in the positive training condition and $n=66$ in the placebo-control condition). To examine generalization of CBM-I to a new task containing new ambiguous information, a $2 \times 2 \times 2 \times 2$ mixed model ANCOVA was conducted on the recognition test data with condition and gender as between-subjects variables, valence (positive vs. negative interpretations) and time (pre- vs. post training) as the within-subject variables, and age as the covariate. Results revealed a significant Condition $\mathrm{x}$ Valence interaction effect, $F(1,134)=4.5, p=0.04, \eta_{\mathrm{p}}{ }^{2}=$ 0.03 , qualified by a significant Condition $\mathrm{x}$ Valence $\mathrm{x}$ Time interaction effect, $F(1,134)=19.6, p<0.001, \eta_{\mathrm{p}}{ }^{2}=0.13$ (see Table 2). No other significant main or interaction effects, including gender and age were observed. The three-way interaction effect was decomposed by analyzing positive and negative interpretations separately. In addition to main 
Table 2 Mean scores on reaction time and recognition interpretive bias task (with standard deviations in parentheses)

\begin{tabular}{|c|c|c|c|c|c|c|c|c|}
\hline & & \multirow[t]{2}{*}{ Valence } & \multicolumn{3}{|c|}{ Positive training } & \multicolumn{3}{|c|}{ Placebo control condition } \\
\hline & & & Negative bias & No bias & Total & Negative bias & No bias & Total \\
\hline \multirow[t]{4}{*}{ Reaction times } & & Positive & $1262(424)$ & $1605(527)$ & $1401(495)$ & $1690(461)^{\mathrm{b}}$ & $1534(508)$ & $1609(489)^{\mathrm{b}}$ \\
\hline & & Negative & $1548(441)$ & $1683(524)$ & $1602(477)^{\mathrm{c}}$ & $1645(447)$ & $1544(455)$ & $1592(465)$ \\
\hline & $\mathrm{T} 1$ & Positive & $1.9(0.3)$ & $2.6(0.3)$ & $2.2(0.5)$ & $2.1(0.4)$ & $2.4(0.4)$ & $2.3(0.4)$ \\
\hline & & Negative & $2.6(0.3)$ & $2.1(0.3)$ & $2.4(0.4)$ & $2.7(0.4)$ & $2.0(0.3)$ & $2.3(0.5)$ \\
\hline \multirow[t]{2}{*}{ Recognition task $\mathrm{k}^{\mathrm{a}}$} & $\mathrm{T} 2$ & Positive & $2.6(0.4)$ & $2.6(0.6)$ & $2.6(0.5)$ & $2.3(0.4)^{\mathrm{b}}$ & $2.5(0.5)$ & $2.4(0.5)^{\mathrm{b}}$ \\
\hline & & Negative & $2.0(0.5)$ & $2.0(0.5)$ & $2.0(0.5)$ & $2.5(0.5)^{\mathrm{b}}$ & $2.3(0.4)$ & $2.4(0.4)^{\mathrm{b}}$ \\
\hline
\end{tabular}

${ }^{\mathrm{a}}$ Recognition ratings ranged from 1 (very different) to 4 (very similar);

${ }^{\mathrm{b}}$ Positive training vs. placebo control condition means differ significantly $(p \leq 0.01)$;

${ }^{\mathrm{c}}$ Positive vs. negative word solution reaction time means differ significantly $(p<0.001)$;

$\mathrm{T} 1$ = before CBM-I training, T2 = after CBM-I training.

effects of Condition (negative interpretation: $F(1,137)=5.2$, $\left.p=0.02, \eta_{\mathrm{p}}{ }^{2}=0.04\right)$ and Time (positive interpretations: $F(1$, $137)=40.1, p<0.001, \eta_{\mathrm{p}}{ }^{2}=0.23$; negative interpretations: $F$ $\left.(1,137)=18.0, p<0.001, \eta_{\mathrm{p}}{ }^{2}=0.12\right)$, significant Condition $\mathrm{x}$ Time interaction effects were observed for both positive and negative interpretations; respectively $F(1,137)=7.2$, $p=0.008, \eta_{\mathrm{p}}{ }^{2}=0.05$ and $F(1,137)=20.8, p<0.001, \eta_{\mathrm{p}}{ }^{2}=0.13$. Before the start of the training, there were no significant differences between the groups in positive, $t(137)=0.8$, $p=0.41, d=0.1$, and negative interpretations, $t(137)=-1.1$, $p=0.27, d=0.2$, as intended. More importantly, after training groups differed significantly in their positive interpretations, $t(137)=-2.5, p=0.01, d=0.4$, and also differed significantly in their negative interpretations, $t(137)=4.6, p<0.001, d=0.8$. That is, positively trained adolescents interpreted new ambiguous information significantly more positively and less negatively than adolescents in the placebo control condition, again indicating successful modification of interpretations and showing generalization to a new task containing new stimuli.

Moderation of Training Effects: The role of pre-training trait anxiety and interpretive bias (Hypothesis 3 )

To investigate differential effects of CBM-I for pre-training level of trait anxiety, an ANOVA was performed in which median split trait anxiety (median $=29.0$ ) was included as a between-subjects factor in the analysis of the reaction time and recognition task data. No significant effects involving trait anxiety were found in the reaction time data ( $p$-values $>0.27$ ), while a significant Valence $\mathrm{x}$ Median split trait anxiety effect was observed in the recognition task data, $F(1,135)=5.8, p=0.02, \eta_{\mathrm{p}}{ }^{2}=0.04$. An independent samples $t$-test revealed that high anxious adolescents interpreted ambiguous information more negative than low anxious adolescents, $t(137)=-2.2, p=0.03, d=0.4$. No other effects involving trait anxiety were significant ( $p$-values> 0.12 ), including any interaction effects with Condition, suggesting that CBM-I was equally effective in high and low trait anxious adolescents.

Similarly, to examine whether pre-training level of negative interpretive bias affected the CBM-I effects, median split interpretive bias (recognition task; median= 0.14 ) was added as a between-subjects factor. Significant effects of pre-existing interpretive bias on CBM-I effects were observed. In the reaction time data, a significant pretraining Interpretive bias (median split) $\mathrm{x}$ Condition $\mathrm{x}$ Valence interaction effect was observed, $F(1,148)=4.2$, $p=0.04, \eta_{\mathrm{p}}{ }^{2}=0.03$ (see Fig. 1 and Table 2). To decompose this interaction effect, we carried out separate analyses for individuals with and without a negative interpretive bias at pre-training. The Condition $x$ Valence interaction effect was only significant in individuals with a negative interpretive bias at the start of the experiment, $F(1,80)=14.5, p<0.001$, $\eta_{\mathrm{p}}{ }^{2}=0.15$ (no negative bias $F(1,68)=0.5, p=0.47, \eta_{\mathrm{p}}{ }^{2}=$ 0.008 ). Within the sub-group of individuals with a negative interpretive bias, it was shown that adolescents who had followed the positive interpretive bias training were significantly faster in solving positive probe word fragments than adolescents who had followed the placebo-control condition, $t(80)=4.4, p<0.001, d=1.0$ (no difference in solving negative word fragments, $t(80)=1.0, p=0.34$, $d=0.2$ ).

When median split interpretive bias (results at pre-test) was added as an additional factor in the recognition task analysis, the following interaction effects involving median split interpretive bias were revealed; Interpretive Bias $\mathrm{x}$ Valence, $F(1,135)=66.7, p<0.001, \eta_{\mathrm{p}}{ }^{2}=0.33$; Interpretive bias $\mathrm{x}$ Valence $\mathrm{x}$ Time, $F(1,135)=49.2, p<0.001, \eta_{\mathrm{p}}{ }^{2}=0.27$; Interpretive bias $\mathrm{x}$ Condition $\mathrm{x}$ Time, $F(1,135)=4.1$, 
Fig. 1 Mean reaction times and standard errors (in ms) to solve positive and negative probes during training depicted for each condition (positive training vs. placebo control condition) and interpretive bias group (pretraining negative bias vs. no negative bias)

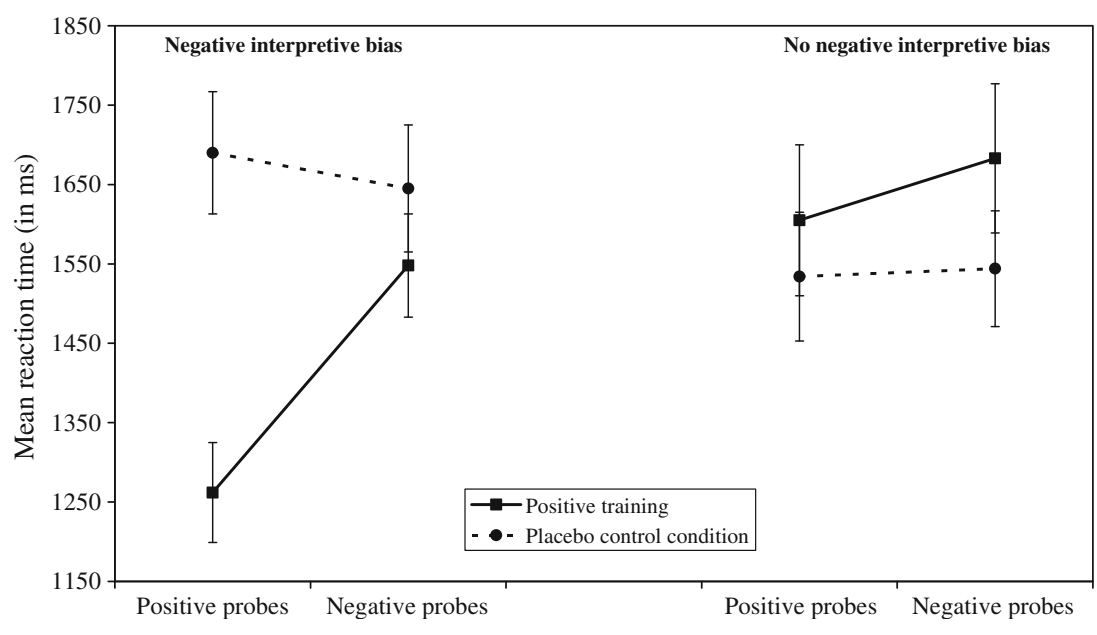

$p=0.04, \eta_{\mathrm{p}}{ }^{2}=0.03$; and most crucially a significant four-way interaction effect: Interpretive bias $\mathrm{x}$ Condition $\mathrm{x}$ Valence $\mathrm{x}$ Time, $F(1,135)=6.1, p=0.02, \eta_{\mathrm{p}}{ }^{2}=0.04$ (see Table 2). Follow-up analyses revealed that training was not effective in modifying interpretations in individuals who had no negative interpretive bias, $F(1,63)=1.8, p=0.19, \eta_{\mathrm{p}}{ }^{2}=0.03$, while training was effective in individuals who had such a negative interpretive bias, Condition x Valence x Time: $F(1,72)=26.4$, $p<0.001, \eta_{\mathrm{p}}{ }^{2}=0.27$. In the group of adolescents with a negative interpretive bias at pre-test, adolescents who had followed the positive training interpreted new ambiguous information significantly more positive, $t(72)=-3.4, p=0.001$, $d=0.8$, and significantly less negative, $t(72)=4.3, p<0.001$, $d=1.0$, than adolescents with a negative interpretive bias at pre-test who had received the placebo-control condition.

In sum, level of trait anxiety did not moderate the effects of training. However, results from both the reaction time data as well as the recognition task data suggested that level of pre-training interpretive bias did moderate those effects. Positive CBM-I appeared to be especially successful for adolescents who had a negative interpretive bias at the start of the experiment.

\section{Effects on State Anxiety (Hypothesis 4)}

To examine the impact of CBM-I on change in state anxiety, a 2 (Condition) x 2 (Time: State anxiety before vs. after training) $\times 2$ (Gender) ANCOVA with age as a covariate was conducted. Results revealed a significant main effect of Gender, $F(1,143)=5.7, p=0.02, \eta_{\mathrm{p}}{ }^{2}=0.04$, and a main effect of Age, $F(1,143)=4.7, p=0.03, \eta_{\mathrm{p}}{ }^{2}=0.03$, reflecting relatively high levels of state anxiety for girls and younger adolescents. The crucial Condition $\mathrm{x}$ Time interaction effect was, however, not significant, $F(1,143)=$ 0.04, $p>0.50, \eta_{\mathrm{p}}{ }^{2}<0.001$, indicating that CBM-I had not affected state anxiety.

\section{Discussion}

The current study was designed to experimentally test whether a Cognitive Bias Modification procedure (CBM) designed to modify threat-related interpretive biases has effects on interpretations and anxiety in adolescents. The adolescent age period is associated with an increase in social anxiety (Westenberg et al. 2007) and it has been argued that interpretive biases play a causal role in (social) anxiety (Beck et al. 1985; Muris and Field 2008). Indeed, there is empirical support that interpretive biases causally influence anxiety in adults (Mathews and Mackintosh 2000), while in children it has been shown that expectancy (interpretive) biases predict anxiety symptoms (Warren et al. 2000). The results of the present study showed that it is possible to modify interpretations of ambiguous social situations in a more positive direction in unselected adolescents. Adolescents trained to interpret information more positively were significantly quicker in solving positive solutions of ambiguity than adolescents in the placebocontrol condition. In addition, when confronted with new ambiguous information presented in a new task, adolescents who followed the CBM-I training interpreted this information more positively and less negatively than adolescents in the control condition. This suggests that the trained interpretive bias generalized to a new task. Subsequent analyses revealed that the CBM-I training was especially effective in adolescents who had a negative interpretive bias. Gender did not moderate the effects of training, which is consistent with earlier findings from CBM-I studies with children (Muris et al. 2008; Vassilopoulos et al. 2009). Meanwhile, gender was associated with both anxiety and interpretive bias; female adolescents were more anxious and had a stronger tendency to interpret ambiguity negatively than male adolescents (see also Muris et al. 2008). Note, that no CBM-I training effects were observed on state anxiety. 
The results concerning the successful modification of interpretations in adolescents are in line with previous findings in unselected children (Muris et al. 2008, 2009) and unselected adults (Mathews and Mackintosh 2000). As no effects on state anxiety were observed, state dependent mood effects are an unlikely explanation for the differential effects on interpretations. The finding that the more vulnerable individuals were especially affected by the modification procedure is also consistent with Muris et al. (2008; but see Muris et al. 2009). However, vulnerable children (i.e. highly anxious) in Muris et al. (2008) were more affected by the negative interpretive bias training; they acquired a stronger negative interpretive bias than low anxious children. Note that the current findings paint a more positive picture for vulnerable adolescents; adolescents with a pre-training threat-related bias were more affected by the positive training than adolescents without a threat-related bias. This is a promising finding in the light of potential application of CBM-I in clinically anxious adolescents. It seems that individuals at risk (either highly anxious or characterized by a negative interpretive bias) are more susceptible to anxiety-relevant influences. While in a context that encourages threat-related cognitions, their malleability might result in stronger maladaptive threat-related cognitions, in a context that encourages non-threatening cognitions, their malleability might result in stronger adaptive cognitions. This line of reasoning is consistent with recent theorizing (differential susceptibility model, Belsky 2005) and gene by environment interaction studies showing that certain children are more affected, both for better and for worse, by their environments (Belsky et al. 2007).

In the current study, CBM-I effects were observed on a recognition task, indicating that the trained interpretive bias generalized to a new task containing new ambiguous stimuli. This is important as it shows that adolescents did not learn a task-specific strategy or a task-specific interpretation style, but that a more general interpretive style was acquired that influenced the processing of new ambiguous information. While the recognition task differs from the training procedure in several aspects, there is also overlap. In both tasks, ambiguous scenarios are presented, explicitly, in a written format, and with sentences appearing one by one. The observed effects on the recognition task could therefore be seen as evidence of close generalization. Future research is necessary to examine whether far generalization of the trained interpretive bias can be observed. Interpretive bias tasks that deviate more from the training procedure could be included in future studies; for example using more visual, pictorial stimuli as faces or film fragments (Amir et al. 2005), or real life social interaction (Voncken and Bögels 2008).

Somewhat unexpectedly, no effects of CBM-I were observed on state anxiety. The positively trained adolescents were not less state anxious after training than the adolescents in the placebo-control condition. This is inconsistent with some of the earlier CBM-I studies in unselected adults (Mathews and Mackintosh 2000; Salemink et al. 2007b; Yiend et al. 2005, Exp 1). However, there are also studies failing to observe the effects on state anxiety in unselected adults (Salemink et al. 2007a; Yiend et al. 2005, Exp. 3). Several explanations for the lack of CBM-I effects on state anxiety can be offered in relation to limitations of the current study. First, absence of CBM-I effects on state anxiety might be related to the fact that state anxiety was assessed with the STAI-C questionnaire, which is a general measure of anxious feelings and sensations. As the training targeted dysfunctional thoughts regarding social situations, the content of training does not match the content of the questionnaire. A match between the training (social anxiety) and the questionnaire could be achieved by measuring specifically social anxiety (for example with the Social Anxiety Scales for children and adolescents (SAS, La Greca 1998).

Second, the lack of CBM-I effects on state anxiety could also be related to the distinction between the concepts of state anxiety and trait anxiety/anxiety vulnerability. Mediation analyses of CBM-I effects have revealed that altered interpretive bias was related to changes in trait anxiety, while the altered interpretive bias was not related to changes in state anxiety (Salemink et al. 2010b). This suggests that it is important to examine CBM-I effects on trait anxiety and not (only) state anxiety. As trait anxiety has been conceptualized as the tendency to react anxiously to a stressor (Bakker et al. 1989; Spielberger 1973), including a stress task containing ambiguity after CBM-I might reveal differential mood effects. Support for this hypothesis has been observed in adults (Mackintosh et al. 2006; Wilson et al. 2006). Participants received a stress task following CBM-I. The stress task consisted of different video clips depicting real-life rescue operations that contained ambiguity, as the outcome of the event remained ambiguous until a clip's final seconds. Results revealed that in both studies, exposure to positive training was associated with less increase in anxiety in response to viewing the stressful accident videos. This is corroborated by findings from cognitive bias modification of attentional bias showing that such a training did not affect state anxiety directly in unselected individuals, but affected stress vulnerability (for children see Eldar et al. 2008; for adults see Macleod et al. 2002).

Third, the lack of CBM-I effects on state anxiety might be related to the role of imagery. Research has shown that mental imagery plays an important role in information processing and emotions (Holmes and Mathews 2005; Holmes et al. 2006). In the current study, participants were instructed to imagine the described situations. However the instructions were minimal and there was no practice in 
vividly imagining the scenarios. Increasing the use of imagery during CBM-I training might be relevant to obtain effects on anxiety (cf. Lothmann et al. 2011).

Fourth, participants were unselected adolescents with an average level of anxious feelings (mean STAI-C trait and state scores between 4th and 6th decile). Compared to highly anxious adolescents, there is less room for CBM-I to reduce anxious feelings. Future studies might add, on the one hand, an assessment of positive emotions and selfesteem as positive CBM training might be associated with an increase in positive feelings (Dandeneau et al. 2007), and on the other hand, CBM-I training might be applied to highly anxious adolescents or clinically anxious adolescents who have higher levels of anxiety and interpretive bias and therefore more room for improvement in both cognitions and mood (Murphy et al. 2007; Vassilopoulos et al. 2009). In addition, participants received a single session of interpretation training. Multiple sessions of training spaced over several days might be necessary for anxiolytic effects to occur (Mathews et al. 2007; Salemink et al. 2009; Vassilopoulos et al. 2009). Future studies could examine the effects of multiple sessions of training and examine the duration of the training effects. The inclusion of booster sessions might optimize effects when results show that effects diminish over time.

Finally, the absence of training effects on anxiety could also be due to the possibility that in adolescents interpretations are not causally related to anxiety (see for example Alfano et al. 2002 for divergent findings regarding the role of cognitions in childhood anxiety). Future research is necessary to investigate more closely the (causal) relationship between anxiety and cognitions in adolescents.

In sum, CBM-I was successful in modifying interpretations in unselected adolescents and stronger effects were observed in individuals who had a threat-related interpretive bias at pretest. These findings are promising when considering training adolescents at risk or adolescents with a clinical diagnosis to interpret ambiguity more positively. Future prevention and intervention trials are necessary to test the long-term clinical and preventative effects of the CBM-I training more rigorously. Note that on-line versions of the training could be developed that could contribute to the problem of long waiting lists and individuals who avoid seeking treatment due to shame or fear of stigmatization. Such a technological innovation could have 24/7 availability and has the potential to be widely disseminated in a way that just is not possible with other types of prevention or intervention programs. However, future studies are required to examine the effects of CBM-I on mood and vulnerability as no effects were observed on state anxiety in the current study.

Acknowledgment We are grateful to Mariëlle Wullaert for help with collecting the data used in this study.
Open Access This article is distributed under the terms of the Creative Commons Attribution Noncommercial License which permits any noncommercial use, distribution, and reproduction in any medium, provided the original author(s) and source are credited.

\section{References}

Alfano, C. A., Beidel, D. C., \& Turner, S. M. (2002). Cognition in childhood anxiety: conceptual, methodological, and developmental issues. Clinical Psychology Review, 22, 1209-1238.

Amir, N., Beard, C., \& Bower, E. (2005). Interpretation bias and social anxiety. Cognitive Therapy and Research, 29, 433-443.

Bakker, F. C., van Wieringen, P. C. W., Van der Ploeg, H. M., \& Spielberger, C. D. (1989). Handleiding bij de Zelfbeoordelings Vragenlijst voor Kinderen (ZBV-K). Een Nederlandse bewerking van de State-Trait Anxiety Inventory for Children (STAI-C). [Manual of the Dutch version of the STAI-C]. Lisse: Swets Test Services.

Beck, A. T., Emery, G., \& Greenberg, R. L. (1985). Anxiety disorders and phobias: A cognitive perspective. New York: Basic Books.

Belsky, J. (2005). Differential susceptibility to rearing influence: An evolutionary hypothesis and some evidence. In B. Ellis \& D. Bjorklund (Eds.), Origins of social mind: Evolutionary psychology and child development (pp. 139-163). New York: Guilford.

Belsky, J., Bakermans-Kranenburg, M. J., \& Van IJzendoorn, M. H. (2007). For better and for worse: differential susceptibility to environmental influences. Current Directions in Psychological Science, 16, 300-304.

Bögels, S. M., \& Zigterman, D. (2000). Dysfunctional cognitions in children with social phobia, separation anxiety disorder, and generalized anxiety disorder. Journal of Abnormal Child Psychology, 28, 205-211.

Cannon, M. F., \& Weems, C. F. (2010). Cognitive biases in childhood anxiety disorders: do interpretive and judgment biases distinguish anxious youth from their non-anxious peers? Journal of Anxiety Disorders, 24, 751-758.

Craske, M. G. (2003). Origins of phobias and anxiety disorders: Why more women than men? Oxford: Elsevier.

Dandeneau, S. D., Baldwin, D. S., Baccus, J. R., Sakellaropoulo, M., \& Pruessner, J. C. (2007). Cutting stress off at the pass: reducing vigilance and responsiveness to social threat by manipulating attention. Journal of Personality and Social Psychology, 93, 651-666.

DeWit, D. J., Ogborne, A., Offord, D. R., \& MacDonald, K. (1999). Antecedents of the risk of recovery from DSM-III-R social phobia. Psychological Medicine, 29, 569-582.

Eldar, S., Ricon, T., \& Bar-Haim, Y. (2008). Plasticity in attention: implications for stress response in children. Behaviour Research and Therapy, 46, 450-461.

Hadwin, J., Frost, S., French, C. C., \& Richards, A. (1997). Cognitive processing and trait anxiety in typically developing children: evidence for an interpretation bias. Journal of Abnormal Psychology, 106, 486-490.

Holmes, E. A., \& Mathews, A. (2005). Mental imagery and emotion: a special relationship? Emotion, 5, 489-497.

Holmes, E. A., Mathews, A., Dalgleish, T., \& Mackintosh, B. (2006). Positive interpretation training: effects of mental imagery versus verbal training on positive mood. Behavior Therapy, 37, 237-247.

La Greca, A. M. (1998). Social anxiety scales for children and adolescents: Manual and instructions for the SASC, SASC-R, $S A S-A$ (adolescents), and parent versions of the scales. Miami: University of Miami, Department of Psychology.

Leitenberg, H., Yost, L. W., \& Carroll-Wilson, M. (1986). Negative cognitive errors in children: questionnaire development, normative 
data, and comparisons between children with and without selfreported symptoms of depression, low self-esteem, and evaluation anxiety. Journal of Consulting and Clinical Psychology, 54, 528536.

Lothmann, C., Holmes, E. A., Chan, S. W. Y., \& Lau, J. Y. F. (2011). Cognitive bias modification training in adolescents: effects on interpretation biases and mood. Journal of Child Psychology and Psychiatry, 52, 24-32.

Mackintosh, B., Mathews, A., Yiend, J., Ridgeway, V., \& Cook, E. (2006). Induced biases in emotional interpretation influence stress vulnerability and endure despite changes in context. Behavior Therapy, 37, 209-222.

MacLeod, C., Rutherford, E., Campbell, L., Ebsworthy, G., \& Holker, L. (2002). Selective attention and emotional vulnerability: assessing the causal basis of their association through the experimental manipulation of attentional bias. Journal of Abnormal Psychology, 111, 107-123.

Mathews, A., \& Mackintosh, B. (2000). Induced emotional interpretation bias and anxiety. Journal of Abnormal Psychology, 109, 602-615.

Mathews, A., \& MacLeod, C. (1994). Cognitive approaches to emotion and emotional disorders. Annual Review of Psychology, $45,25-50$

Mathews, A., Ridgeway, V., Cook, E., \& Yiend, J. (2007). Inducing a benign interpretational bias reduces trait anxiety. Journal of Behavior Therapy and Experimental Psychiatry, 38, 225-236.

Miers, A. C., Blöte, A. W., Bögels, S. M., \& Westenberg, P. M. (2008). Interpretation bias and social anxiety in adolescents. Journal of Anxiety Disorders, 22, 1462-1471.

Muris, P., \& Field, A. P. (2008). Distorted cognition and pathological anxiety in children and adolescents. Cognition and Emotion, 22, 395-421.

Muris, P., Huijding, J., Mayer, B., \& Hameetman, M. (2008). A space odyssey: experimental manipulation of threat perception and anxiety-related interpretation bias in children. Child Psychiatry and Human Development, 39, 469-480.

Muris, P., Huijding, J., Mayer, B., Remmerswaal, D., \& Vreden, S. (2009). Ground control to Major Tom: experimental manipulation of anxiety-related interpretation bias by means of the "space odyssey" paradigm and effects on avoidance tendencies in children. Journal of Anxiety Disorders, 23, 333-340.

Murphy, R., Hirsch, C. R., Mathews, A., Smith, K., \& Clark, D. M. (2007). Facilitating a benign interpretation bias in a high socially anxious population. Behaviour Research and Therapy, 45, 15171529.

Salemink, E., van den Hout, M. A., \& Kindt, M. (2007a). Trained interpretive bias and anxiety. Behaviour Research and Therapy, 45, 329-340

Salemink, E., van den Hout, M. A., \& Kindt, M. (2007b). Trained interpretive bias: validity and effects on anxiety. Journal of Behavior Therapy and Experimental Psychiatry, 38, 212-224.
Salemink, E., van den Hout, M. A., \& Kindt, M. (2009). Effects of positive interpretive bias modification in highly anxious individuals. Journal of Anxiety Disorders, 23, 676-683.

Salemink, E., van den Hout, M. A., \& Kindt, M. (2010a). Generalisation of modified interpretive bias across tasks and domains. Cognition and Emotion, 24, 453-464.

Salemink, E., van den Hout, M. A., \& Kindt, M. (2010b). How does Cognitive Bias Modification affect anxiety? Mediation analyses and experimental data. Behavioural and Cognitive Psychotherapy, 38, $59-66$.

Spielberger, C. D. (1973). Preliminary test manual for the State-Trait Anxiety Inventory for Children. Palo Alto: Consulting Psychologists Press.

Vassilopoulos, S. P., Banerjee, R., \& Prantzalou, C. (2009). Experimental modification of interpretation bias in socially anxious children: changes in interpretation, anticipated interpersonal anxiety, and social anxiety symptoms. Behaviour Research and Therapy, 47, $1085-1089$.

Voncken, M. J., \& Bögels, S. M. (2008). Social performance deficits in social anxiety disorder: reality during conversation and biased perception during speech. Journal of Anxiety Disorders, 22, 1384-1392.

Warren, S. L., Emde, R. N., \& Sroufe, L. A. (2000). Internal representations: predicting anxiety from children's play narratives. Journal of the American Academy of Child and Adolescent Psychiatry, 39, 100-107.

Weems, C. F., Berman, S. L., Silverman, W. K., \& Saavedra, L. M. (2001). Cognitive errors in youth with anxiety disorders: the linkages between negative cognitive errors and anxious symptoms. Cognitive Therapy and Research, 25, 559-575.

Weems, C. F., Costa, N. M., Watts, S. E., Taylor, L. K., \& Cannon, M. F. (2007). Cognitive errors, anxiety sensitivity, and anxiety control beliefs: their unique and specific associations with childhood anxiety symptoms. Behavior Modification, 31, 174-201.

Westenberg, P. M., Gullone, E., Bokhorst, C. L., Heyne, D. A., \& King, N. J. (2007). Social evaluation fear in childhood and adolescence: normative developmental course and continuity of individual differences. British Journal of Developmental Psychology, 25, 471-483.

Wilson, E. J., MacLeod, C., Mathews, A., \& Rutherford, E. M. (2006). The causal role of interpretive bias in anxiety reactivity. Journal of Abnormal Psychology, 115, 103-111.

Wittchen, H. U., \& Fehm, L. (2003). Epidemiology and natural course of social fears and social phobia. Acta Psychiatrica Scandinavica, 108, 4-18.

Woodward, L. J., \& Fergusson, D. M. (2001). Life course of young people with anxiety disorders in adolescence. Journal of the American Academy of Child and Adolescent Psychiatry, 40, 1086-1093.

Yiend, J., Mackintosh, B., \& Mathews, A. (2005). Enduring consequences of experimentally induced biases in interpretation. Behaviour Research and Therapy, 43, 779-797. 